ARTICLE OPEN

\title{
Cytochrome $b d$ promotes Escherichia coli biofilm antibiotic tolerance by regulating accumulation of noxious chemicals
}

\author{
Connor J. Beebout (iD), Levy A. Sominsky ${ }^{1}$, Allison R. Eberly ${ }^{1,4}$, Gerald T. Van Horn ${ }^{1}$ and Maria Hadjifrangiskou (iD ${ }^{1,3 凶}$
}

Nutrient gradients in biofilms cause bacteria to organize into metabolically versatile communities capable of withstanding threats from external agents including bacteriophages, phagocytes, and antibiotics. We previously determined that oxygen availability spatially organizes respiration in uropathogenic Escherichia coli biofilms, and that the high-affinity respiratory quinol oxidase cytochrome $b d$ is necessary for extracellular matrix production and biofilm development. In this study we investigate the physiologic consequences of cytochrome $b d$ deficiency in biofilms and determine that loss of cytochrome bd induces a biofilmspecific increase in expression of general diffusion porins, leading to elevated outer membrane permeability. In addition, loss of cytochrome $b d$ impedes the proton mediated efflux of noxious chemicals by diminishing respiratory flux. As a result, loss of cytochrome bd enhances cellular accumulation of noxious chemicals and increases biofilm susceptibility to antibiotics. These results identify an undescribed link between $E$. coli biofilm respiration and stress tolerance, while suggesting the possibility of inhibiting cytochrome $b d$ as an antibiofilm therapeutic approach.

npj Biofilms and Microbiomes (2021)7:35; https://doi.org/10.1038/s41522-021-00210-x

\section{INTRODUCTION}

Biofilms are multicellular bacterial communities commonly encountered in the environment and during infection. Because bacteria in biofilms are intrinsically resistant to a variety of stressors, including antibiotics, phagocytes, and bacteriophages, the ability to form biofilms is a critical bacterial survival strategy ${ }^{1-7}$. Accordingly, the vast majority of bacteria in the environment and in the human body-up to 80 percent according to recent estimates ${ }^{8}$-are believed to exist in the biofilm state. Due to the stress tolerance of biofilms and the lack of biofilm-specific therapies, a biofilm associated infection typically necessitates chronic suppressive antibiotic treatment or surgical removal of the infected material ${ }^{9}$. Improvements in antibiofilm therapeutics are critically needed and would greatly advance our ability to reduce infection burden.

Within biofilms, bacteria consume or alter chemicals as they diffuse through the community, thus generating a variety of nutrient gradients that ensure individual bacteria are exposed to highly variable local environmental conditions ${ }^{3}$. This environmental heterogeneity induces bacteria to differentiate into metabolically distinct, and oftentimes cooperative, subpopulations, which enhance the overall resilience and versatility of the community $^{2,3,10-12}$. We previously hypothesized that the presence of oxygen gradients in biofilms would generate differentially respiring subpopulations, each of which uniquely contributes to biofilm development. Indeed, our work with uropathogenic $E$. coli (UPEC) - the primary cause of urinary tract infections and one of the most common human bacterial pathogens ${ }^{13-15}$-indicates that differential oxygen availability across biofilm regions leads to heterogenous expression of respiratory enzymes, with the aerobic quinol oxidases being the most abundantly expressed ${ }^{16}$.

$E$. coli is a facultative anaerobe that encodes a modular electron transport chain containing a multitude of interchangeable dehydrogenases, quinol electron carriers, and terminal oxidases/ reductases ${ }^{17,18}$. This architecture provides $E$. coli an enormous degree of metabolic flexibility, allowing bacteria to colonize diverse niches. Despite being a facultative anaerobe, previous studies establish that UPEC requires aerobic respiration during infection and to form biofilms ${ }^{10,16,19-23}$. During bladder infection, UPEC consumes amino acids, which feed into the TCA cycle to energize the aerobic respiratory chain ${ }^{19,20,22}$. UPEC encodes three aerobic respiratory quinol oxidases: one proton pumping hemecopper oxidase, cytochrome bo, and two non-proton pumping bdtype oxidases, cytochrome bd and cytochrome $b d_{2}^{17,24}$. Cytochrome bo is a low oxygen affinity quinol oxidase transcriptionally and biochemically optimized for use under atmospheric oxygen tensions ${ }^{25-27}$. By contrast, the $b d$-type oxidases have high oxygen affinity and are optimized for use under low oxygen tensions ${ }^{25-27}$. Importantly, the $b d$-type oxidases also provide resistance against oxidative and nitrosative stress, suggesting these enzymes may play a critical role in enhancing stress tolerance under microaerobic conditions encountered in biofilms and the urinary $\operatorname{tract}^{28-30}$.

Given the importance of aerobic respiration to UPEC pathogenesis and biofilm formation, as well as the oxygen regulated transcriptional control of quinol oxidases, we previously investigated the spatial distribution of respiration in biofilms ${ }^{16}$. In agreement with studies in Pseudomonas aeruginosa, we determined that expression of the two most abundant oxidases, cytochrome $b d$ and bo, is inversely correlated in the community along the biofilm oxygen gradient, suggesting $E$. coli biofilms contain differentially respiring subpopulations ${ }^{16,31}$. We then sought to disentangle the contributions of each quinol oxidase to biofilm physiology. Surprisingly, despite robust expression of all three aerobic quinol oxidases, only loss of cytochrome $b d$ has any significant effect on biofilm development. Cytochrome bd deficiency induces severe architectural disturbances in biofilms and reduces their ability to prevent external stressors from

\footnotetext{
${ }^{1}$ Department of Pathology, Microbiology, and Immunology, Vanderbilt University Medical Center, Nashville, TN, USA. ${ }^{2}$ Vanderbilt University, Nashville, TN, USA. ${ }^{3}$ Vanderbilt Institute for Infection, Immunology, and Inflammation, Vanderbilt University Medical Center, Nashville, TN, USA. ${ }^{4}$ Present address: Division of Clinical Microbiology, Department of Laboratory Medicine and Pathology, Mayo Clinic, Rochester, MN, USA. ${ }^{\circledR}$ email: maria.hadjifrangiskou@vumc.org
} 
entering the biomass ${ }^{16}$. Deletion of the locus that encodes cytochrome bd leads to upregulation of the low-affinity oxidase cytochrome bo and impairs biofilm development without compromising ATP levels ${ }^{16}$. This study established the presence of differentially respiring subpopulations in $E$. coli biofilms, and argues respiratory heterogeneity is a fundamental contributor to biofilm physiology.

In this work we aimed to determine how cytochrome bd expressing biofilm subpopulations contribute to $E$. coli biofilm physiology. To do so, we interrogated and compared the cellular physiology of cytochrome bd-deficient cells in the planktonic and biofilm state. We determine that loss of cytochrome bd increases the abundance of multiple outer membrane proteins in biofilm cells, including general diffusion porins responsible for antibiotic uptake. Consequently, cytochrome bd-deficient biofilm cells have increased outer membrane permeability and more readily take up noxious chemicals from the environment. In addition to enhancing cellular uptake of noxious chemicals, loss of cytochrome $b d$ impairs their efflux by impeding the proton dependent activity of resistance-nodulation-division (RND) efflux pumps and possibly other tripartite export proteins. As a result, loss of cytochrome bd increases biofilm susceptibility to multiple clinically relevant antibiotics. Interestingly, this increased sensitivity is a biofilmspecific phenomenon, as deletion of cytochrome $b d$ has no effect on antibiotic susceptibility in planktonic cells. This study reveals a previously undescribed link between respiration and biofilm stress tolerance in $E$. coli and suggests the possibility of inhibiting cytochrome bd as a therapeutic strategy for preventing and treating urinary tract infections.

\section{RESULTS}

\section{Loss of cytochrome bd increases biofilm antibiotic sensitivity}

We previously determined that uropathogenic Escherichia coli (UPEC) exhibits marked respiratory heterogeneity in biofilms, and that loss of cytochrome bd-but not other respiratory quinol oxidases-induces significant disruptions to biofilm development ${ }^{16}$. Furthermore, we demonstrated that these disruptions are solely attributable to cytochrome bd deficiency, as extrachromosomal complementation of $\triangle c y d A B$ with a plasmid encoding the $C y d A B X$ operon under native transcriptional control fully rescues the observed biofilm deficits ${ }^{16}$. Based on these observations, we hypothesized that cytochrome $b d$ is necessary for the formation of metabolically versatile biofilm communities capable of withstanding antibiotics and other external stressors. To test this, we first evaluated the effects of antibiotics on biofilms formed by the well-characterized uropathogenic Escherichia coli cystitis isolate UTI89 and an isogenic mutant strain lacking cytochrome bd $(\triangle c y d A B)^{16}$.

A recent meta-analysis demonstrates that measuring biofilm antimicrobial susceptibility using a single method or a single drug concentration is often inadequate due to a high degree of variability between methods ${ }^{32}$. As such, we sought to investigate the susceptibility of $\triangle c y d A B$ biofilms antibiotic susceptibility across a range of conditions. First, we grew polyvinyl chloride (PVC)associated biofilms for $48 \mathrm{~h}$, treated with a panel of antibiotics for another $72 \mathrm{~h}$, and measured overall biofilm abundance by the crystal violet assay ${ }^{33}$. Treatment of wild-type biofilms with supralethal doses of $\beta$-lactams (ampicillin), aminoglycosides (gentamicin), or fluoroquinolones (ciprofloxacin) led to a $40-75 \%$ reduction in total biomass but did not eradicate the biofilm, highlighting the resilience of biofilms in the face of our current therapeutic strategies (Fig. 1a). After normalizing biomass to the untreated control of each strain, we determined both strains have similar relative reductions in biomass after treatment with $\beta$-lactams or fluoroquinolones, but $\triangle c y d A B$ biofilms are significantly more susceptible to aminoglycosides than the parental strain (Fig. 1a).

To more thoroughly define biofilm sensitivity to antibiotics and more closely approximate antibiotic concentrations encountered by bacteria under clinically relevant conditions, we grew PVCassociated biofilms as previously described and treated with decreasing concentrations of antibiotics (Fig. 1b-d). Interestingly, at lower $\beta$-lactam concentrations we observe minimal effect on total biomass, and $\triangle c y d A B$ biofilms exhibit a small, but statistically significant increase in biomass relative to wild-type (Fig. 1b). By contrast, $\triangle c y d A B$ biofilms are significantly more sensitive to both aminoglycosides and fluoroquinolones across the range of concentrations tested (Fig. 1c, d). This effect was most pronounced at lower antibiotic concentrations similar to serum antibiotic concentrations achieved clinically.

Although the crystal violet assay represents a convenient method of assessing biofilm biomass, it does not provide meaningful insights into biofilm architecture or physiology ${ }^{34}$. To characterize the structural effect of antibiotics on $\triangle c y d A B$ biofilms, we grew biofilms on PVC coverslips, treated with antibiotics as described above, and imaged the biofilms using confocal laser scanning microscopy (Fig. 1e). As expected, antibiotic treatment had relatively minor effects on the structural characteristics of wild-type biofilms (Fig. 1e). Whereas $\beta$-lactam treatment led to topographic changes in wild-type biofilms without affecting the apparent density of cells, aminoglycoside or fluoroquinolone treatment had minimal effect (Fig. 1e). By contrast, treatment with all three classes of antibiotics led to widespread structural disruption of $\triangle C y d A B$ biofilms and reduced cell density (Fig. 1e), grossly consistent with the reductions in biomass observed by crystal violet assays.

The above data indicate that antibiotic treatment induces a more significant loss of biomass in $\triangle c y d A B$ biofilms as compared to those biofilms formed by the wild-type strain. To determine whether the antibiotic-induced biomass reductions are caused by increased cell death, we quantified percent survival of biofilm cells after $24 \mathrm{~h}$ of antibiotic treatment in a colony biofilm model ${ }^{35}$. Treatment of wild-type biofilms with $\beta$-lactams had no significant effect on biofilm CFUs, whereas treatment with aminoglycosides or fluoroquinolones led to significant reductions in CFUs per biofilm (Fig. 1f). When compared to wild-type, $\triangle c y d A B$ biofilms had significantly reduced cell survival after treatment with $\beta$-lactams (91 and 49 percent survival in UTI89 and $\triangle c y d A B$, respectively) and aminoglycosides ( 25 and $10 \%$ survival in UTI89 and $\triangle c y d A B$, respectively), indicating $\triangle c y d A B$ biofilm cells are more sensitive to antibiotic-induced cell death (Fig. 1f). By contrast, $\triangle c y d A B$ biofilm cells are somewhat less sensitive to fluoroquinolone treatment than wild-type ( 2 and 8 percent survival in UTI89 and $\triangle c y d A B$, respectively) (Fig. 1f). In total, despite some expected variability between experimental approaches ${ }^{32}$, these data demonstrate that loss of cytochrome bd renders biofilms more susceptible to the clinically important $\beta$-lactam, aminoglycoside, and fluoroquinolone classes of antibiotics.

\section{Cytochrome bd does not affect planktonic susceptibility to antibiotics}

Our results thus far indicate that cytochrome $b d$ affects the ability of biofilms to withstand antibiotics. To determine whether cytochrome bd influences antibiotic sensitivity in the planktonic state, we performed broth microdilution assays to measure the minimum inhibitory concentration (MIC) of ampicillin, gentamicin, and ciprofloxacin against each strain (Fig. 2a). Surprisingly, we observe no significant differences in MIC between strains for ampicillin and ciprofloxacin, and a small increase in MIC for $\triangle c y d A B$ for gentamicin $(1.8$ and $2.9 \mu \mathrm{g} / \mathrm{mL}$ for UTI89 and $\triangle c y d A B$, respectively). These MIC values are similar to previously reported values for UTI89, although we observe a somewhat elevated MIC 
a

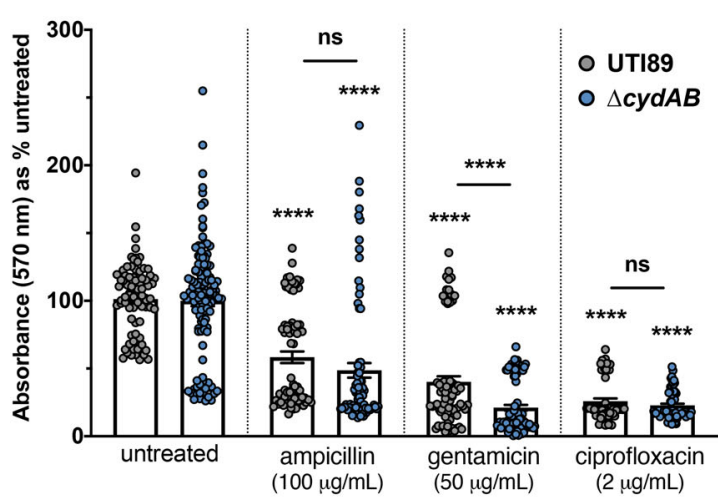

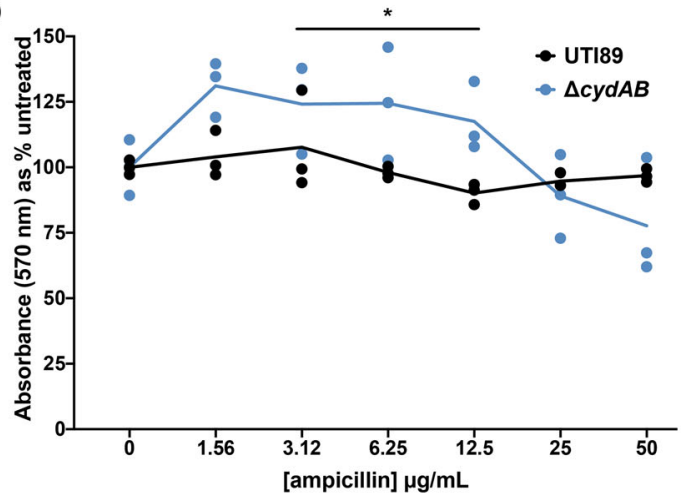

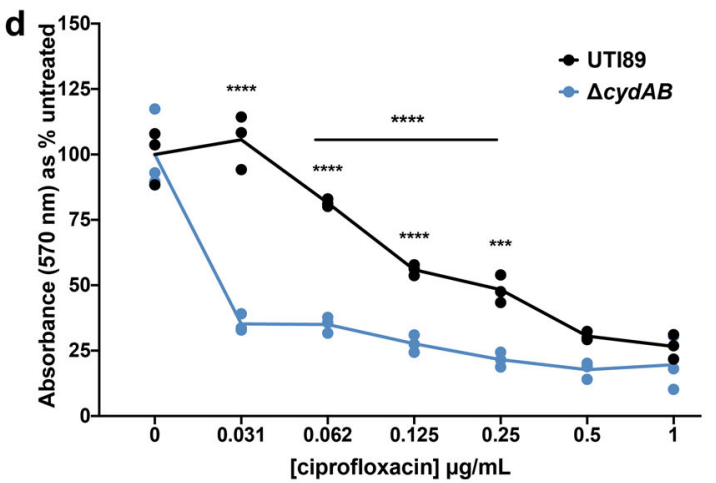

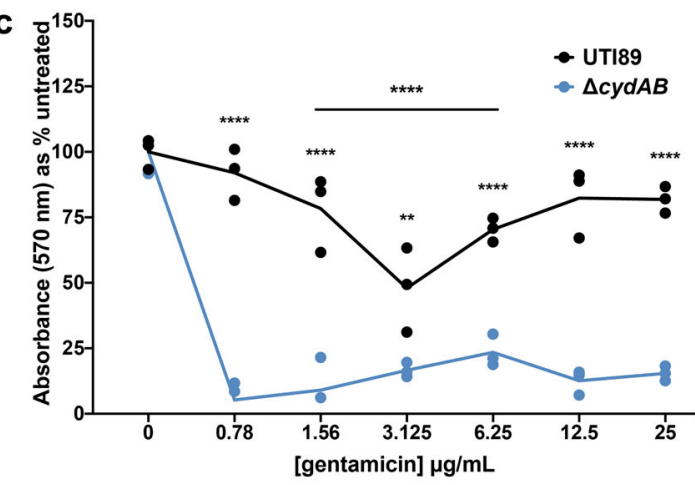
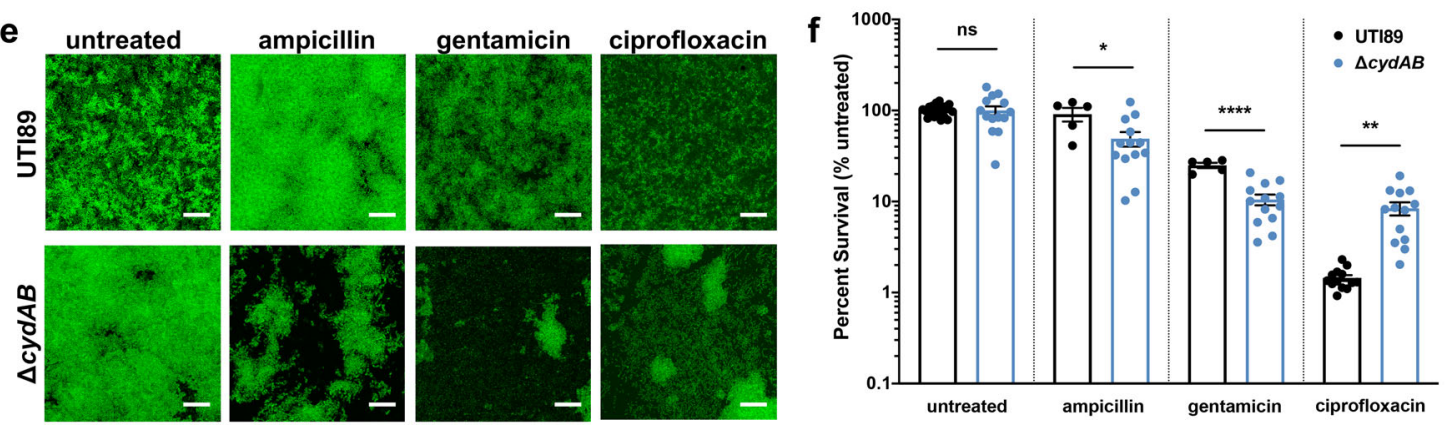

Fig. 1 Loss of cytochrome bd increases biofilm antibiotic sensitivity. a PVC-associated air-liquid interface biofilms were grown for $48 \mathrm{~h}$, treated with antibiotics for $72 \mathrm{~h}$, and biomass was quantified using the crystal violet assay. Biofilm biomass was quantified for wild-type UTI89 and isogenic cytochrome $b d$-deficient strain $\triangle c y d A B$. Data were normalized to the untreated control of each strain. The mean absorbance value in each treatment group was compared to the untreated control of the same strain (asterisks), and to the mean value of the other strain in the same treatment group (horizontal line with asterisks) using a two-tailed Welch's $t$-test. Data represent at least three biological replicates with at least eight technical replicates each. Each dot represents an independent well. $\mathbf{b}$-d Dose response curves depicting the total biomass of biofilms after treatment with decreasing concentrations of ampicillin (b), gentamicin (c), or ciprofloxacin (d). Data were analyzed using a two-way ANOVA to evaluate overall differences between strain across the range of concentrations tested (horizontal line with asterisks), with multiple comparisons used to evaluate differences in mean at each concentration (asterisks). Data represent three biological replicates with three technical replicates each. Each dot represents the mean value of a biological replicate. Solid lines connect mean values at each concentration. e Representative images of antibiotic treated biofilms stained with STYO9 and imaged by confocal laser scanning microscopy. At least five images were acquired along the air-liquid interface of three biological replicates. Scale bar is $20 \mu \mathrm{m}$. $\mathbf{f}$ Survival of bacteria in colony biofilms after antibiotic treatment. Colony biofilms were grown on YESCA agar for $72 \mathrm{~h}$, and biofilms were transferred to a new plate with or without antibiotics. After $24 \mathrm{~h}$ of antibiotic treatment, biofilms were homogenized and plated to enumerate CFUs. Mean values in each treatment group were statistically compared using a two-tailed unpaired $t$-test. Data are representative of at least five biological replicates. Each dot represents a biological replicate. For all graphs, data are presented as mean \pm SEM. ${ }^{*} p<0.05,{ }^{* *} p<0.01,{ }^{* * *} p<0.001,{ }^{* * * *} p<0.0001$.

for ampicillin as compared to previous studies ${ }^{36}$. Next, to assess antibiotic sensitivity across a range of clinically relevant antibiotics, we performed disk diffusion assays according to Clinical \& Laboratory Standards Institute (CLSI) guidelines and procedures followed by the clinical microbiology laboratory at Vanderbilt University Medical Center ${ }^{37}$. These analyses revealed that $\triangle c y d A B$ had a slightly larger zone of inhibition for most antibiotics tested (median percent difference: 6.5 percent; range: 0-13 percent) (Fig. $2 \mathrm{~b}$ and Supplementary Table 1$). \triangle c y d A B$ had a significantly larger zone of inhibition after treatment with six antibiotics: meropenem, cefazolin, ceftazidime, aztreonam, sulfamethoxazole-trimethoprim, and nitrofurantoin (Fig. 2b, underlined). Of note, two of these antibiotics-sulfamethoxazole-trimethoprim and nitrofurantoinare first line treatments for urinary tract infections ${ }^{38}$. Interestingly, we do not observe differences in sensitivity to gentamicin using disk diffusion assays despite the observed increase in MIC, highlighting the effects of distinct growth conditions (static liquid culture versus solid agar surface) on metabolism and antibiotic susceptibility. Although clinical guidelines and CLSI breakpoints would deem both strains equally susceptible to antibiotics 
a
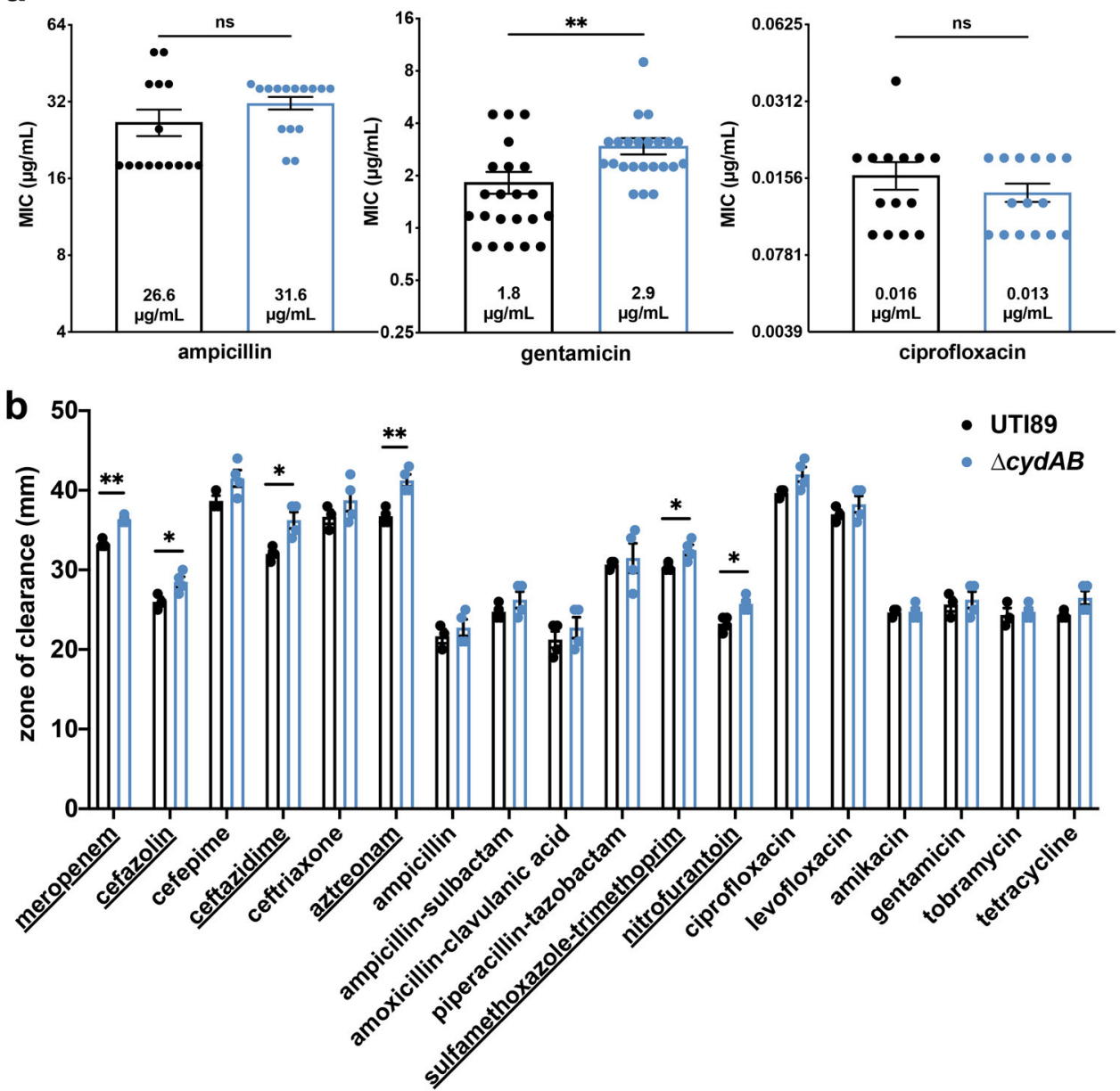

Fig. 2 Cytochrome bd has minimal effect on planktonic antibiotic susceptibility. a Graph depicting minimum inhibitory concentration (MIC) for ampicillin, gentamicin, and ciprofloxacin. Data are representative of at least six biological replicates. Data were analyzed using a twotailed unpaired $t$-test. $\mathbf{b}$ Graph depicting the zone of inhibition from disk diffusion assays. Data are representative of at least three biological replicates. Each dot represents a biological replicate. Mean values were statistically compared using two-tailed Welch's $t$-test. Underlined antibiotics attained statistical significance. For all panels, data are presented as mean \pm SEM. ${ }^{*} p<0.05,{ }^{* *} p<0.01,{ }^{* * *} p<0.001,{ }^{* * * *} p<0.0001$.

(Supplementary Table 1$)^{37}$, these analyses reveal a small but consistent trend toward increased antibiotic susceptibility in $\triangle c y d A B$, which may be indicative of metabolic derangements also present in the planktonic state.

To determine whether the observed changes in antibiotic sensitivity are physiologically relevant, we performed time kill kinetics assays to measure the rate of antibiotic-induced cell death in planktonic cultures (Fig. 3). Cultures were grown to midlogarithmic phase, split in two, and one flask was treated with antibiotics at $5 \times$ the minimal inhibitory concentration. The survival curves were analyzed using a two-way ANOVA to statistically compare the rate of antibiotic-induced cell death between strains over time. To fully evaluate the potential role of cytochrome $b d$ in planktonic antibiotic sensitivity, these assays were performed on wild-type, $\triangle c y d A B$, and a strain that encodes cytochrome $b d$ as its sole aerobic quinol oxidase $(\triangle a p p B C \triangle c y o A B)$. These assays revealed no statistically significant differences in the rate of antibiotic killing between strains after treatment with $\beta$-lactams, aminoglycosides, fluoroquinolones, or a clinically relevant synergistic combination of $\beta$-lactams and aminoglycosides (Fig. 3a-d). We observe a high degree of variability in survival during the early time points (15-30 $\mathrm{min}$ ) of cultures treated with aminoglycosides as compared with other antibiotics, raising the possibility that there is heterogeneous early response to aminoglycoside treatment (Fig. 3b, d). These results may reflect differences in antibiotic uptake or efficacy resulting from alterations in the electron transport chain composition and proton motive force. In total, these results indicate that, despite small changes in antibiotic susceptibility observed between strains, loss of cytochrome $b d$ has no significant or clinically relevant effect on antibiotic susceptibility in the planktonic state. These results are in agreement with recent work in K-12 E. coli demonstrating that loss of $c y d B$ has no discernible effect on planktonic sensitivity to reactive oxygen species or aminoglycosides ${ }^{39}$. In combination with previous data, these results demonstrate that loss of cytochrome $b d$ specifically increases bacterial susceptibility to antibiotics in the biofilm state.

\section{Loss of cytochrome bd alters the outer membrane of biofilm cells}

Our data indicate that loss of cytochrome bd increases biofilm sensitivity to antibiotics without significantly affecting planktonic sensitivity. In principle, such a biofilm-specific effect could be caused by changes to the extracellular matrix, outer membrane, or cellular metabolism. In our previous work, we reported that $\triangle c y d A B$ biofilms have changes to the abundance, composition, and organization of the extracellular matrix, suggesting that changes in the extracellular matrix may play a role in antibiotic tolerance by influencing community organization and modulating antibiotic sequestration $^{16}$. In this study, we investigate how 

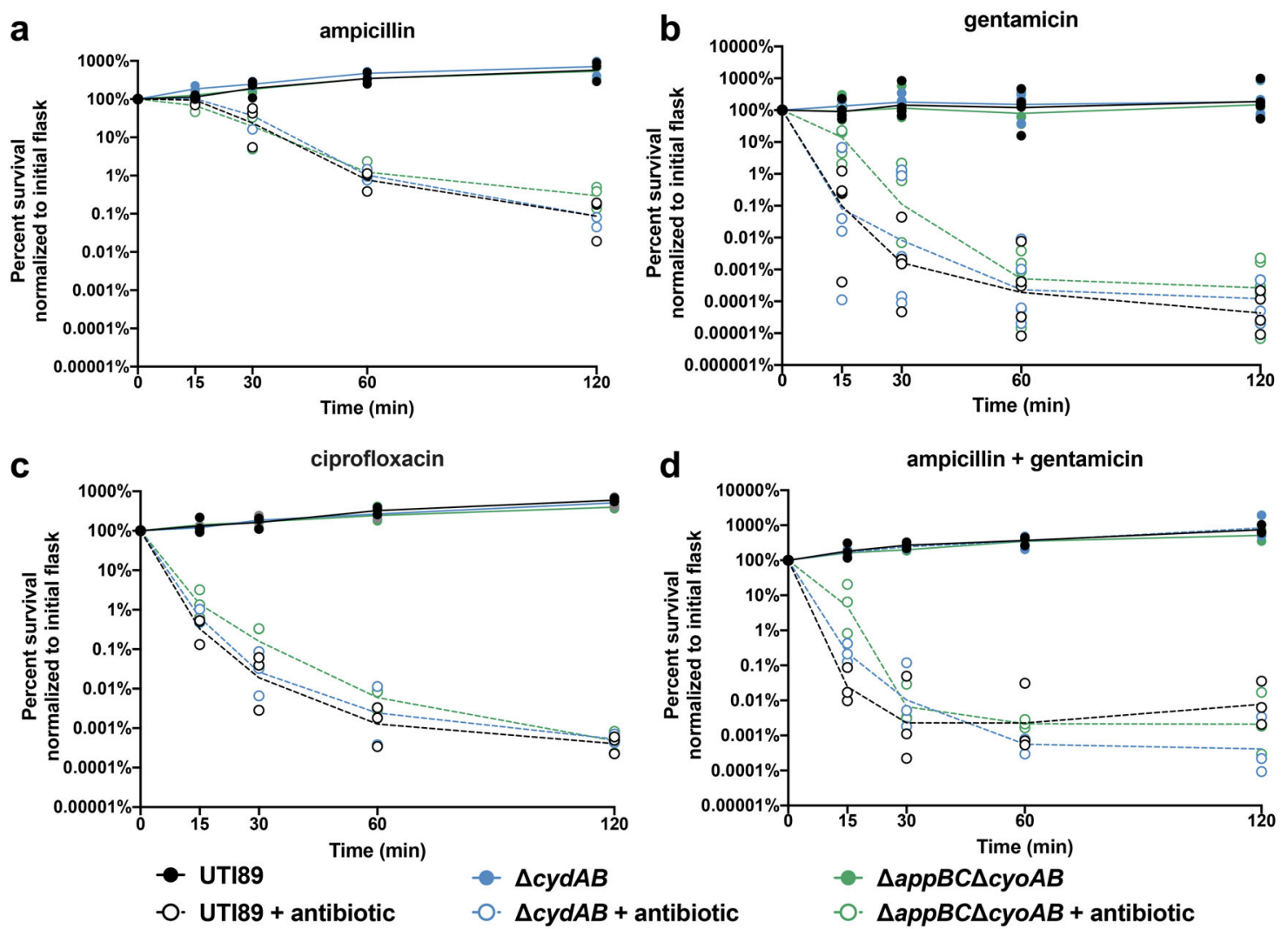

Fig. 3 Cytochrome bd does not affect the rate of antibiotic-induced cell death in planktonic cells. a-d Time kill kinetics assays were performed to evaluate the susceptibility of UTI89 (black), $\triangle c y d A B$ (blue), and $\triangle a p p B C \triangle c y o A B$ (green) to ampicillin (a), gentamicin (b), ciprofloxacin (c), or a combination of ampicillin and gentamicin (d) at 5x MIC. Data were analyzed by a two-way ANOVA with Tukey's multiple comparisons test and determined to have no significant differences between strains. Data are representative of at least three biological replicates. Lines connect geometric mean at each time point. Each dot represents a biological replicate.

cytochrome bd impacts biofilm antibiotic tolerance by influencing the outer membrane and cellular energetics.

Prior to import into the cell, antibiotics must interact with and traverse the negatively charged outer membrane. This step is particularly important for charged antibiotics such as cationic aminoglycosides. As such, one possible explanation for the alterations in antibiotic efficacy is that $\triangle c y d A B$ cells have changes to the charge of their outer membrane with consequent effects on antibiotic import. To test this possibility, we measured the interaction of equine cytochrome $c$ with the outer membrane of biofilm and planktonic cells ${ }^{40}$. Cytochrome $c$ is a polycationic molecule known to interact electrostatically with the negatively charged bacterial cell envelope ${ }^{40}$. Quantifying the binding of cytochrome $c$ to the cell envelope can be used as a proxy for determining the relative charge of the cell envelope, as described previously ${ }^{40}$. In planktonic cells, we observe less cytochrome $c$ binding to the outer membrane (63 percent of wild-type), suggesting wild-type cells have a more negatively charged outer membrane than $\triangle c y d A B$ (Fig. 4). However, in biofilm cells, no significant difference in cytochrome $c$ binding is observed between wild-type and $\triangle c y d A B$ (Fig. 4) suggesting the outer membrane has a similar charge in both strains. These results indicate that changes to outer membrane charge cannot explain the altered antibiotic sensitivity of $\triangle c y d A B$ biofilm cells.

We next sought to investigate how loss of cytochrome bd influences outer membrane permeability. To do so, we extracted the outer membrane and extracellular matrix from colony biofilms using established methods and performed liquid chromatography tandem mass spectrometry (LC-MS/MS) (Fig. 5a and Supplementary Table 2$)^{41}$. These experiments identified 30 outer membrane associated or secreted proteins with significantly altered

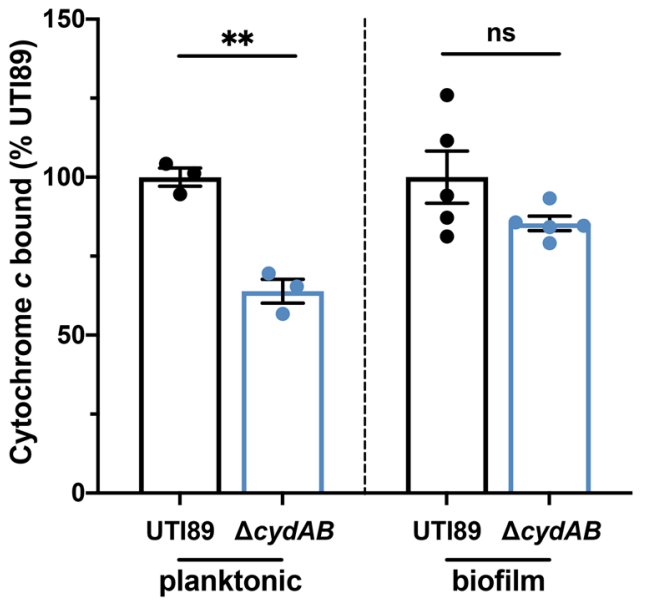

Fig. 4 Outer membrane charge is unaffected in $\triangle$ cydAB biofilm cells. Outer membrane charge was investigated by measuring the binding of cationic cytochrome $c$ to planktonic and biofilm cells. Data were analyzed by a two-tailed unpaired $t$-test. Data are representative of at least three biological replicates, and are presented as mean \pm SEM. Each dot represents a biological replicate. ${ }^{*} p<0.05,{ }^{* *} p<0.01,{ }^{* * *} p<0.001,{ }^{* * * *} p<0.0001$.

abundance between wild-type and $\triangle c y d A B$ (defined as fold change $\geq 1.5$ and $p<0.05$ ) (Fig. 5a and Supplementary Table 2). Of these, two proteins are of significantly decreased abundance in $\triangle c y d A B$ biofilms, and 28 proteins are of significantly increased abundance. Notably, seven of the 28 proteins with increased 

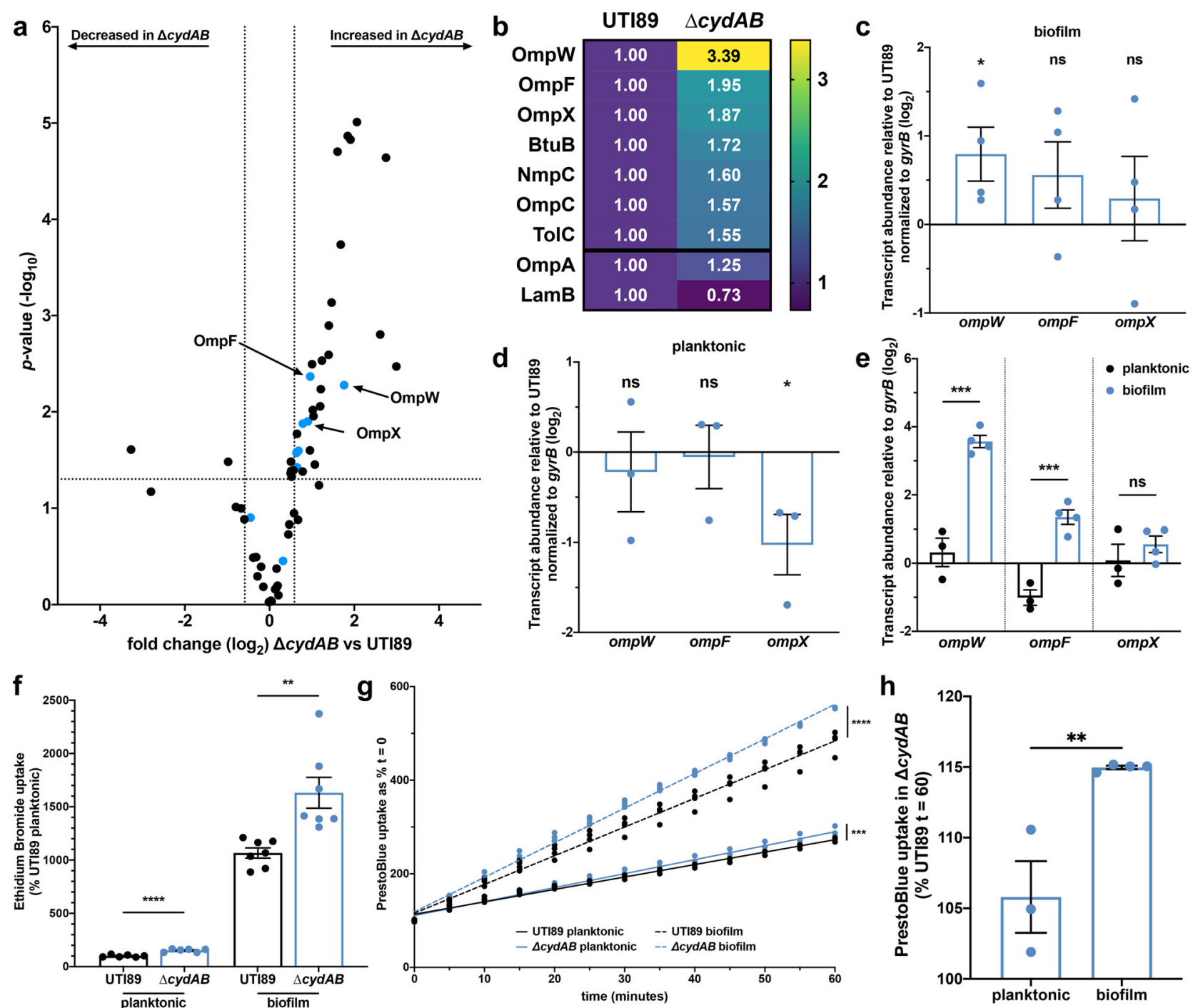

Fig. 5 Cytochrome bd-deficient biofilm cells have elevated uptake of noxious chemicals. a Volcano plot depicting all outer membrane or secreted proteins detected by LC-MS/MS performed on outer membrane and extracellular matrix extracts. Blue dots represent outer membrane channel proteins. b Heatmap depicting the relative difference in abundance of outer membrane proteins in UTI89 and $\triangle c y d A B$. Each cell contains fold difference in abundance relative to UTI89. OmpA and LamB did not attain statistical significance. Data in a, $\mathbf{b}$ are representative of three biological replicates per strain. c-e RT-qPCR was performed to determine the relative fold difference in outer membrane protein transcript abundance normalized to gyrB abundance between UTI89 and $\triangle c y d A B$ in samples derived from homogenized colony biofilms grown on YESCA agar for 11 days (c) and planktonic cells (d). e Difference in outer membrane protein transcript abundance between $\triangle c y d A B$ biofilm and planktonic cells was evaluated by comparing the abundance of each transcript to gyrB abundance. Data in c-e are representative of four biological replicates and were analyzed using a two-tailed unpaired $t$-test. $\mathbf{f}$ Cellular uptake of membrane impermeant ethidium bromide into planktonic cells and cells extracted from homogenized biofilms. Data are representative of at least six biological replicates and were analyzed by a two-tailed unpaired $t$-test. $\mathbf{g}$ Cellular uptake of resazurin-based dye PrestoBlue into planktonic cells (solid lines) and cells extracted from homogenized biofilms (dashed lines) was quantified over time. Data were fit to a linear regression model and analyzed by statistically comparing the slope. Data are representative of at least three biological replicates. $\mathbf{h}$ Percent difference in PrestoBlue uptake at $60 \mathrm{~min}$ in $\triangle c y d A B$ cells as compared to UTI89. Data were analyzed with a two-tailed unpaired $t$-test. Except where noted all data are presented as mean \pm SEM, and each dot represents a biological replicate. ${ }^{*} p<0.05,{ }^{* *} p<0.01,{ }^{* * *} p<0.001,{ }^{* * * *} p<0.0001$.

abundance in $\triangle c y d A B$ are outer membrane channel proteins responsible for the uptake of environmental compounds (Fig. 5a, b, blue dots and Supplementary Table 2), suggesting $\triangle c y d A B$ biofilm cells may have a more permeable outer membrane. In particular, two proteins with significantly elevated abundance$\mathrm{OmpF}$ and $\mathrm{OmpC}$ - are classical general diffusion porins responsible for the nonspecific uptake of hydrophilic small molecules including $\beta$-lactams, fluoroquinolones, and aminoglycosides ${ }^{42-44}$.

To validate the mass spectrometry results, we performed RTqPCR on RNA extracted from whole, homogenized colony biofilms. Based on the finding that outer membrane channel proteins are significantly more abundant in $\triangle c y d A B$ biofilms, in combination with the known role for this class of proteins in antibiotic uptake, we chose to measure transcript abundance of the three outer membrane proteins with the most increased abundance in $\triangle c y d A B$ (OmpW, OmpF, and OmpX) (Fig. 5b). Consistent with the proteomics data, RT-qPCR revealed that $\triangle c y d A B$ biofilms have significantly more abundant ompW transcript (1.9-fold greater than wild-type), and elevated abundance of both ompF and ompX transcript (1.6 and 1.4-fold greater than wild-type, respectively), albeit below the threshold of significance (Fig. 5c). To determine if the observed increase in omp transcript is biofilm specific, we performed RT-qPCR targeting the same genes with RNA derived from planktonic cultures (Fig. 5d). Interestingly, 
in planktonic cells we observe no significant difference in ompW and $o m p F$ abundance between strains, and significantly decreased ompX transcript in $\triangle c y d A B$ (Fig. 5d). Finally, we compared abundance of each transcript between $\triangle c y d A B$ planktonic and biofilm cells and observe that $\triangle c y d A B$ biofilm cells have significantly elevated steady state transcript of ompW and ompF as compared to $\triangle c y d A B$ planktonic cells (Fig. 5e). These results are in agreement with previous studies in K-12 E. coli demonstrating that several outer membrane proteins, including $\mathrm{OmpC}, \mathrm{OmpF}$, and $\mathrm{NmpC}$, have elevated expression in biofilms relative to planktonic cultures ${ }^{45}$. Together these results indicate that cytochrome bd-deficient cells have elevated expression of several outer membrane proteins in the biofilm state. Guided by these results, we next sought to investigate how loss of cytochrome bd influences cellular accumulation of noxious chemicals.

\section{Cytochrome bd-deficient biofilm cells have enhanced uptake of noxious chemicals}

Because outer membrane proteins serve as the primary site of cellular entry for hydrophilic small molecules, the increased abundance of outer membrane proteins suggests that $\triangle c y d A B$ biofilm cells may have a more permissive outer membrane, and therefore increased uptake of antibiotics and other noxious chemicals. To test this, we measured accumulation of ethidium bromide into planktonic cells as well as cells extracted from colony biofilms (Fig. 5f). Ethidium bromide is outer membrane impermeant and fluoresces after intercalation into DNA. In planktonic cells we observe a small, but statistically significant increase in ethidium bromide accumulation in $\triangle c y d A B$. In biofilm cells, ethidium bromide uptake is significantly elevated for both strains and highest in $\triangle c y d A B$ biofilm cells ( 10- and 16-fold elevated in UTI89 and $\triangle C y d A B$, respectively), consistent with the observed increase in outer membrane protein abundance in biofilms (Fig. $5 e, f)$. These data demonstrate that biofilm cells have elevated outer membrane permeability, higher uptake of noxious chemicals as compared to planktonic cells, and that loss of cytochrome $b d$ enhances cellular uptake of these compounds.

The increased ethidium bromide accumulation represents a net increase in uptake and could be explained by alterations to the rate of influx, efflux, or both. To differentiate these possibilities, we quantified influx kinetics using resazurin-based PrestoBlue, a dye that becomes fluorescent after import into the cytosol ${ }^{46}$. In planktonic cells we observe a small, but statistically significant increase in the rate of dye influx in $\triangle c y d A B$ relative to wild-type (slope: 2.6 and 3.0 for wild-type and $\triangle c y d A B$, respectively) (Fig. 5g). Consistent with ethidium bromide uptake data, in biofilm cells we observe a significant increase in the rate of accumulation for both strains (Fig. 5f, g). In addition to the overall increase in influx, we observe a significantly increased rate of influx in $\triangle c y d A B$ biofilm cells as compared to wild-type biofilm cells (slope: 6.1 and 7.4 for wild-type and $\triangle c y d A B$, respectively) (Fig. $5 \mathrm{~g}$ ). In biofilm cells, the maximal fluorescence value obtained after 60 min was 15 percent higher in $\triangle c y d A B$ compared to wild-type-an $\sim 2.5$-fold greater difference than that observed in planktonic cells (six percent) (Fig. $5 \mathrm{~h})$. Together these results indicate that biofilm cells have an elevated rate of influx compared to planktonic cells, and that loss of cytochrome bd enhances cellular influx of noxious compounds.

\section{Loss of cytochrome bd impairs efflux of noxious chemicals in biofilm cells}

In addition to affecting the rate of antibiotic influx, loss of cytochrome $b d$ may also influence the efficiency of efflux. To test this, we measured the rate of ethidium bromide efflux from wildtype and $\triangle c y d A B$ cells. Cells were loaded with ethidium bromide under energy-limited conditions, and efflux was monitored over time using fluorescence based methods (Fig. 6a, b) ${ }^{47}$. Cells were either left in energy-limited conditions as a control for passive decay in signal, or re-energized by the addition of glucose ${ }^{47}$. The data were fit to a one phase decay model, and differences between strains were determined by statistically comparing the best fit models.

We first measured the rate of efflux in planktonic cells (Fig. 6a). As expected, the slowest decay in signal was observed in a mutant strain lacking $a c r A B$, the resistance-nodulation-division (RND) efflux pump primarily responsible for efflux of ethidium bromide (Fig. $6 a)^{47}$. The rate of efflux was significantly elevated in both wild-type and $\triangle C y d A B$ as compared to $\triangle a c r A B$ (UTI89 vs $\triangle a c r A B$ : $p=0.002 ; \triangle c y d A B$ vs $\triangle a c r A B: p<0.0001$ ) (Fig. 6a). Surprisingly, the rate of efflux was significantly elevated in $\triangle c y d A B$ as compared to wild-type $(p=0.02)$ (Fig. 6a), indicating loss of cytochrome bd enhances efflux under aerobic conditions in planktonic cells.

Next, we homogenized colony biofilms, removed the extracellular matrix, and extracted cells to measure the rate of ethidium bromide efflux in biofilm cells (Fig. 6b). As expected, $\triangle a c r A B$ biofilm cells again have the slowest rate of efflux, and the rate of efflux did not significantly differ between $\triangle a c r A B$ planktonic and biofilm cells $(p=0.23)$ (Fig. 6b). Consistent with data from planktonic cells, we observe a significantly elevated rate of efflux in wild-type biofilm cells as compared to $\triangle a c r A B$ biofilm cells ( $p<$ 0.0001 ) (Fig. 6b). The rate of efflux did not significantly differ between wild-type planktonic and biofilm cells $(p=0.53)$. Strikingly, the rate of efflux in $\triangle c y d A B$ biofilm cells was indistinguishable from $\triangle a c r A B$ biofilm cells $(p=0.45)$ (Fig. 6b), indicating that loss of cytochrome bd functionally inactivates efflux in biofilm cells. $\triangle c y d A B$ biofilm cells have a significant reduction in efflux both compared to $\triangle c y d A B$ planktonic cells $(p<0.0001)$ and compared to wild-type biofilm cells $(p=0.0005)$. Although $\triangle a c r A B$ biofilms exhibit some minor structural anomalies relative to wildtype, they are morphologically distinct from $\triangle c y d A B$ biofilms, suggesting a lack of $A c r A B$ efflux activity does not fully explain the biofilm developmental defects observed in $\triangle c y d A B$ (Supplementary Fig. 1). These results indicate that loss of cytochrome $b d$ impairs efflux of noxious chemicals in a biofilm-specific manner.

\section{Loss of cytochrome bd impairs respiratory flux and diminishes the proton motive force}

The impaired efflux in $\triangle c y d A B$ biofilm cells could be explained by changes in the expression, abundance, or activity of efflux pumps. To investigate these possibilities, we performed RT-qPCR on samples derived from planktonic and biofilm cells to measure the abundance of $a c r B$ transcript. Interestingly, $a c r B$ transcript is $\sim 50$ fold more abundant in biofilm cells relative to planktonic cells, and we observe no significant difference in abundance between wildtype and $\triangle c y d A B$ (Fig. $6 \mathrm{c}$ ). Additionally, our proteomics results reveal no significant difference in AcrA abundance between strains (fold change 0.99 in UTI89 compared to $\triangle c y d A B, p=0.95$ ). These data argue the impaired efflux in $\triangle c y d A B$ biofilm cells is not explainable by changes in $a c r A B$ expression or abundance; rather, loss of cytochrome $b d$ appears to reduce the activity of AcrABand potentially other proton dependent tripartitate exporters-in biofilm cells.

Because cytochrome $b d$ is a respiratory quinol oxidase that contributes to electron flow and the establishment of the proton motive force, we hypothesized that loss of cytochrome bd would impair respiratory flux, diminish the proton motive force, and consequently impair the proton mediated efflux through RND, major facilitator superfamily (MFS), and multidrug and toxin extrusion (MATE) family transporters ${ }^{48}$. To test this hypothesis, we quantified respiratory flux using triphenyl tetrazolium chloride (TTC) reduction assays (Fig. $6 \mathrm{~d}, \mathrm{e})$. $\Pi \mathrm{C}$ is a redox sensitive dye that undergoes an irreversible color change upon reduction by NADH dehydrogenase and is commonly used as an indicator of respiratory activity ${ }^{31}$. Cells lacking cytochrome bd $(\triangle c y d A B)$ displayed significantly diminished overall TTC reduction as 

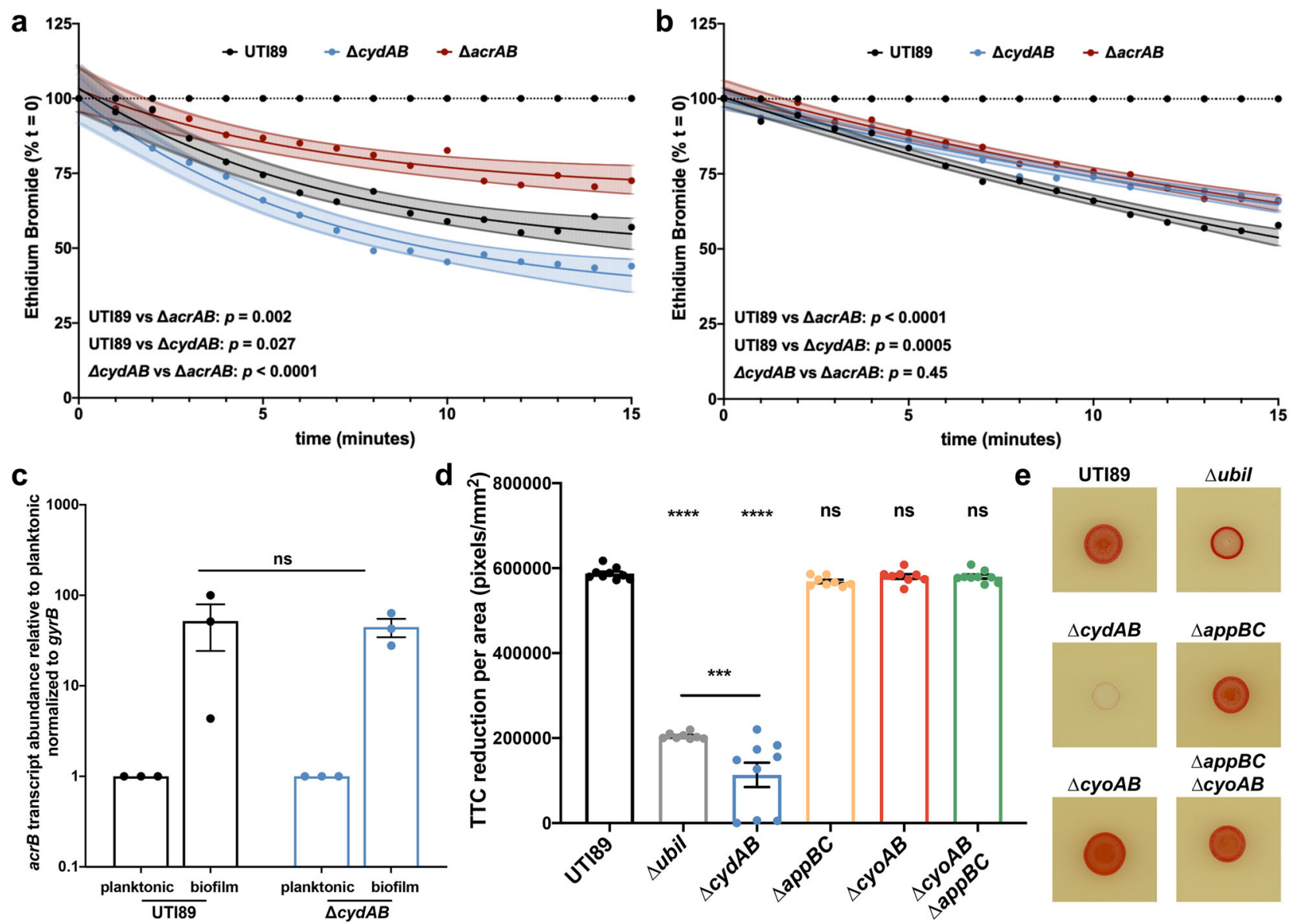

Fig. 6 Loss of cytochrome bd impairs efflux by diminishing respiratory flux. $\mathbf{a}$, b Efflux of ethidium bromide was measured in planktonic cells (a) and cells extracted from homogenized colony biofilms (b). Data were fit to a one phase decay model, and statistical comparisons were made between the curve of best fit for each strain. Data are presented as mean \pm 95 percent confidence interval. c RT-qPCR was performed to measure $a c r B$ transcript abundance normalized to gyrB abundance in UTI89 and $\triangle c y d A B$ planktonic and biofilm cells. Mean values were statistically compared with a two-tailed unpaired $t$-test. Data are presented as mean \pm SEM, and each dot represents a biological replicate. d, e Respiratory flux was quantified in wild-type, $\Delta u$ bil (ubiquinone synthase mutant), and quinol oxidase mutants by measuring triphenyl tetrazolium chloride (TTC) reduction. d Quantification of TTC reduction per unit area in spot colonies. Data were analyzed by a one-way ANOVA with Dunnett's multiple comparisons test. Data are presented as mean \pm SEM, and each dot represents a biological replicate. e Representative images of TTC reduction assays. Red color indicates respiratory activity. Data in a-c are representative of three biological replicates, and data in $\mathbf{d}$, e are representative of at least eight biological replicates. ${ }^{*} p<0.05,{ }^{* *} p<0.01,{ }^{* * *} p<0.001,{ }^{* * * *} p<0.0001$.

compared to wild-type, indicating that loss of cytochrome bd diminishes flux through the electron transport chain and impairs the generation of the proton motive force (Fig. $6 \mathrm{~d}$, e). Importantly, the diminished $T T C$ reduction in $\triangle c y d A B$ is rescued by extrachromosomal complementation with a plasmid encoding the $c y d A B X$ operon under native transcriptional control (Supplementary Fig. 2$)^{16}$. Despite the observed impairments in respiratory flux, we observe no significant reduction in ATP levels in $\triangle c y d A B$ colony biofilms (Supplementary Fig. 3), consistent with our previous reports ${ }^{16}$. This suggests that these cells likely are not fermenting, as under fermentative conditions the $\mathrm{F}_{0} \mathrm{~F}_{1}$-ATPase is reversed and consumes ATP to generate a proton gradient ${ }^{49}$. Rather, the reduced respiratory flux is likely due to inefficient respiration in hypoxic biofilm regions where cytochrome $b d$ is most highly expressed ${ }^{16}$. Consistent with this, loss of cytochrome bo or $b d_{2}$ did not significantly impact $\Pi \mathrm{C}$ reduction, arguing that cytochrome $b d$ is the dominant respiratory enzyme under the conditions tested and its loss cannot be compensated for by expression of other quinol oxidases (Fig. $6 \mathrm{~d}$, e). Together these data argue that loss of cytochrome bd impedes efflux of noxious chemicals by disrupting respiratory flux and impairing the proton dependent activity of efflux pumps.

\section{DISCUSSION}

Living in spatially structured biofilm communities affords resident bacteria significant protection from a wide array of exogenous threats, including antibiotics and host immune defenses. While this has been demonstrated in several different studies, the composite mechanisms that lead to resilience in the biofilm remain largely uncharacterized. In this work, we build upon our previous findings that revealed a spatial organization in quinol oxidase expression in $E$. coli biofilms ${ }^{16}$. Here, we expand our understanding of how cytochrome bd expression influences biofilm-specific resistance to antibiotics. We demonstrate that loss of cytochrome bd increases antibiotic susceptibility in a biofilm-specific manner by regulating the cellular accumulation of antibiotics and other noxious chemicals. This enhanced accumulation results from a combination of increased abundance of general diffusion porins and decreased efficiency of proton mediated efflux. Consistent with these findings, alterations in the expression or activity of porins and efflux pumps is a common contributor to antibiotic resistance in clinical isolates ${ }^{42,44,50}$.

We report that disrupting cytochrome $b d$-mediated respiration in uropathogenic $E$. coli leads to a general enhancement of antibiotic susceptibility specifically when bacteria are found in the 
biofilm state. A likely explanation for the distinct antibiotic susceptibility phenotypes observed between $\triangle c y d A B$ planktonic and biofilm cells is that $E$. coli encodes a highly flexible respiratory chain-allowing bacteria to adapt to loss of cytochrome bd by altering the expression of other oxidases ${ }^{17,24}$-and that the spatial organization of biofilms imparts unique metabolic constraints not encountered in well-mixed planktonic cultures. Indeed, we previously reported that expression of cytochrome bo was increased nearly tenfold in $\triangle c y d A B$ biofilms, indicating that rather than simply impeding respiration, loss of cytochrome bd forces bacteria to instead transition to respiring via cytochrome $b o^{16}$. Such a transition likely has minimal effect in shaking, logarithmic phase planktonic cultures, where all cells are expected to have access to near atmospheric levels of oxygen, as cytochrome bo is a low-affinity respiratory quinol oxidase optimized for use under atmospheric oxygen tensions ${ }^{17,24}$. In fact, because it is a proton pumping oxidase, cytochrome bo is more energetically efficient than cytochrome $b d\left(\mathrm{H}^{+} / \mathrm{e}^{-}\right.$ratio: 2 and 1 for cytochrome bo and $b d$, respectively), potentially explaining the increased efflux observed in $\triangle c y d A B$ planktonic cells. In spatially structured biofilms, by contrast, most cells are exposed to subatmospheric oxygen levels ${ }^{3}$. As such, in these cells cytochrome bd is the dominant respiratory enzyme, and the low oxygen affinity of cytochrome bo ensures that simply overexpressing this oxidase cannot compensate for lack of cytochrome $b d$. As a result of the unique biochemistries of these respiratory oxidases and the spatially structured nature of biofilms, loss of cytochrome bd reduces the efficiency of respiration and proton mediated efflux in a biofilm-specific manner.

While our results stand in contrast to the antibiotic resistance phenotype generated by respiratory deficiency in small colony variants described in Staphylococcus aureus and other species ${ }^{51}$, they are consistent with studies in Mycobacterium tuberculosis in which targeting of the electron transport chain is a promising avenue for the development of novel classes of antibiotics ${ }^{52,53}$. In recent years two electron transport chain inhibitors-bedaquiline, an ATPase inhibitor, and pretomanid, a nitric oxide donor and respiratory poison-have been approved by the Food and Drug Administration (FDA) for the treatment of multidrug resistant $M$. tuberculosis ${ }^{52,54}$. Additionally, promising results from a phase 2 clinical trial were recently reported for telacebec (Q203), an inhibitor of the terminal cytochrome oxidase supercomplex $b c_{1}$ $a a_{3}{ }^{55}$. In addition to the known clinical utility of these agents, preclinical studies have identified numerous small molecule inhibitors of all electron transport chain components (NADH dehydrogenases, succinate dehydrogenases, quinol oxidases, ATPase), some of which are known to eradicate even highly drug resistant isolates ${ }^{52,53}$. Although the potential for disrupting energetics as a therapeutic approach has not been thoroughly evaluated outside mycobacterial species, in combination with our findings these studies raise the possibility of inhibiting cytochrome $b d$ to treat or prevent urinary tract infections. Notably, previous work has identified several natural small molecule quinol analogs that serve as potent inhibitors of $E$. coli quinol oxidases, including several molecules that preferentially inhibit bd-type oxidases $^{52,56}$. Importantly, the bd-type oxidases are unique to bacteria, suggesting inhibitors of cytochrome bd could be used clinically to inhibit biofilm formation and potentiate the effects of antibiotics. Together, this work reveals that the spatial stratification of respiration is a fundamental driver of $E$. coli biofilm stress tolerance and suggest the possibility of rewiring the electron transport chain as an antibiofilm therapeutic approach.

\section{METHODS}

\section{Bacterial strains and growth conditions}

All studies were performed in uropathogenic Escherichia coli cystitis isolate UTI89 ${ }^{57}$ and isogenic deletion mutants. For all analyses, strains were propagated from a single colony in Lysogeny broth (LB) (Fisher) at pH 7.4 overnight at $37^{\circ} \mathrm{C}$ with shaking unless otherwise noted. Genetically manipulated strains were created using $\lambda$-red recombinase system ${ }^{58}$. Quinol oxidase mutant strains, ubil mutant strains, and $\triangle c y d A B$ complementation constructs were created in previous studies ${ }^{16,22}$. Primers used for gene deletions and qPCR are listed in Supplementary Table 3.

\section{Crystal violet biofilm assays}

Determination of biofilm biomass was performed using the crystal violet assay as previously described ${ }^{33}$. Overnight cultures were diluted to optical density $600 \mathrm{~nm}\left(\mathrm{OD}_{600}\right)=0.05$, and $100 \mu \mathrm{L}$ of the diluted culture was aliquoted into a 96-well polyvinyl chloride (PVC) plate (Costar). Plates were incubated in a humid chamber at room temperature, washed and stained with crystal violet, and disaggregated using 35 percent acetic acid. For antibiotic assays, biofilms were grown for $48 \mathrm{~h}$, antibiotics or vehicle was added to each well, and biofilms were grown another $72 \mathrm{~h}$ prior to determining biomass. Total biomass was determined by measuring the absorbance at $570 \mathrm{~nm}$ using a SpectraMax i3 microplate reader (Molecular Devices).

\section{Microscopy}

Plastic coverslips (Fisher) were placed in the diluted culture in a six well plate and allowed to grow $48 \mathrm{~h}$ at room temperature. Antibiotics were added at $48 \mathrm{~h}$, and biofilms were allowed to grow another $72 \mathrm{~h}$ before being fixed in 4\% PFA and stained with SYTO 9 (ThermoFisher). Images were taken using a Zeiss 710 confocal laser scanning microscope. To obtain a representative sample of the biofilms, at least three images were taken along the air-liquid interface from at least five biological replicates.

\section{Biofilm survival assays}

Colony biofilm survival assays were performed as previously described ${ }^{35}$. Briefly, $10 \mu \mathrm{L}$ overnight culture was spotted on a piece of filter paper covered in a thin layer of $1.2 \times$ yeast extract casamino acids (YESCA) agar and allowed to incubate at room temperature. After $72 \mathrm{~h}$, biofilms were transferred to new YESCA agar with or without antibiotics. After $24 \mathrm{~h}$ of treatment, biofilms were homogenized by two rounds of vortexing and sonication, serially diluted, and plated to enumerate colony forming units (CFUs).

\section{Disk diffusion assays}

Antimicrobial susceptibility testing was performed using the Kirby-Bauer disk diffusion method. Testing was performed on 5\% Mueller-Hinton agar using commercially available antimicrobial disks (BD) according to Clinical \& Laboratory Standard Institute (CLSI) guidelines, M100-ed $30^{37}$. The following disks were used for antimicrobial susceptibility testing: meropenem $(10 \mu \mathrm{g})$, cefazolin $(30 \mu \mathrm{g})$, cefepime $(30 \mu \mathrm{g})$, ceftazidime $(30 \mu \mathrm{g})$, ceftriaxone $(30 \mu \mathrm{g})$, aztreonam $(30 \mu \mathrm{g})$, ampicillin $(10 \mu \mathrm{g})$, ampicillin-sulbactam $(10 / 10 \mu \mathrm{g})$, amoxicillin-clavulanic acid $(20 / 10 \mu \mathrm{g})$, piperacillin-tazobactam $(100 / 10 \mu \mathrm{g})$, trimethoprim-sulfamethoxazole $(1.25 / 23.75 \mu \mathrm{g})$, nitrofurantoin $(300 \mu \mathrm{g})$, ciprofloxacin $(5 \mu \mathrm{g})$, levofloxacin $(5 \mu \mathrm{g})$, amikacin $(30 \mu \mathrm{g})$, gentamicin $(10 \mu \mathrm{g})$, tobramycin $(10 \mu \mathrm{g})$, and tetracycline $(30 \mu \mathrm{g})$.

\section{Broth microdilution assays}

Broth microdilution assays were performed to determine the minimum inhibitory concentration (MIC), as previously described ${ }^{59}$. Briefly, antibiotics were serially diluted two-fold in a 96-well plate. To increase the precision of the MIC estimate, two independent dilution series were used for each antibiotic. Overnight cultures were diluted to $\mathrm{OD}=0.06$ in Mueller-Hinton Broth (BD Difco), and $100 \mu \mathrm{L}$ was added to each well. Cultures were incubated overnight at $37^{\circ} \mathrm{C}$, and MIC was determined by assessing the row at which visible growth of bacteria was inhibited.

\section{Time kill kinetics assay}

Overnight cultures were subcultured in $20 \mathrm{~mL}$ Mueller-Hinton Broth (BD Difco) to an $\mathrm{OD}_{600}=0.05$ and grown 3-4 $\mathrm{h}$ to mid-logarithmic phase. Each strain was normalized to an $\mathrm{OD}_{600}=0.5$ in PBS and split into two flasks. One flask of each strain was inoculated with antibiotic to a final concentration at five times the MIC of wild-type; the other flask served as an untreated control. After addition of the antibiotic, $100 \mu \mathrm{L}$ of culture 
was removed from each flask at each time point $(0,15,30,60$, and $120 \mathrm{~min})$ for CFU enumeration.

\section{RT-qPCR}

RNA was extracted from day 11 colony biofilms or planktonic cultures using the RNeasy kit (Qiagen). RNA was DNase treated using Turbo DNase I (Invitrogen), and reverse transcribed using SuperScript III Reverse Transcriptase (Invitrogen). cDNA was amplified in an Applied Biosystems StepOne Plus Real-Time PCR machine using SYBR green and primers listed in Table S3. All reactions were performed using CDNA from at least three biological replicates. Each reaction was performed in triplicate with at least two different CDNA concentrations. A melt curve analysis was performed using genomic DNA and for every reaction with CDNA to verify primer specificity. Relative fold difference in transcript abundance was determined using the $\Delta \Delta C_{\mathrm{T}}$ method $^{60}$. Transcripts were normalized to $g y r B$ abundance.

\section{Cytochrome c binding assay}

Outer membrane charge was assessed by measuring the amount of cationic cytochrome $c$ binding to cells, as previously described ${ }^{40}$. Planktonic cells were extracted from mid-logarithmic phase cultures. Biofilm cells were extracted from homogenized colony biofilms grown for 11 days on YESCA agar. Cells were normalized to $\mathrm{OD}=2.0$ and washed twice in $20 \mathrm{mM}$ MOPS ( $\mathrm{pH}$ 7.0). Cationic equine cytochrome $c$ (Sigma) was added to $0.5 \mathrm{mg} / \mathrm{mL}$. Cells were incubated with cytochrome $c$ for $10 \mathrm{~min}$ at room temperature. After incubation, cells were pelleted by centrifugation, and unbound cytochrome $c$ was measured from the supernatant by quantifying absorption at $530 \mathrm{~nm}$.

\section{Proteomics}

Outer membrane and extracellular matrix samples were extracted as described previously ${ }^{41}$. Biofilms were grown for 11 days on $1.2 \times$ YESCA agar containing $40 \mu \mathrm{g} / \mathrm{mL}$ Congo Red. Biofilms were homogenized in cold $10 \mathrm{mM}$ Tris- $\mathrm{HCl} \mathrm{pH} 7.4$ using an Omni Tissue Homogenizer five times for one minute per cycle. To increase yield and robustness, each sample is a pooled collection of 100 individually grown biofilms. Data are representative of three pooled replicates per strain. The homogenate was centrifuged three times for $10 \mathrm{~min}$ at $5000 \times g$ to remove cells. $\mathrm{NaCl}$ was added to the supernatant (final concentration $170 \mathrm{mM}$ ) and centrifuged for one hour at $13,000 \times g$ to pellet the ECM. The ECM pellet was washed in $10 \mathrm{mM}$ Tris- $\mathrm{HCl}$ $\mathrm{pH} 7.4$ with $4 \%$ SDS and incubated at room temperature rocking overnight. Twenty five microgram of protein was precipitated by adding $1 / 3$ volume of 100 percent $w / v$ trichloroacetic acid (TCA). After washing two times with ice cold acetone, the protein pellet was resuspended in $8 \mathrm{M}$ urea $100 \mathrm{mM}$ tris $\mathrm{pH} 8.5$, reduced using TCEP, alkylated with iodoacetamide, diluted back to $2 \mathrm{M}$ urea and digested with $0.5 \mathrm{ug}$ of trypsin overnight at $37^{\circ} \mathrm{C}$. Resulting peptides were analyzed by high resolution data dependent LCMS/MS. Briefly, peptides were autosampled onto a $200 \mathrm{~mm}$ by $0.1 \mathrm{~mm}$ (Jupiter 3 micron, $300 \mathrm{~A}$ ), self-packed analytical column coupled directly to a Q-exactive plus mass spectrometer (ThermoFisher) using a nanoelectrospray source and resolved using an aqueous to organic gradient. Both the intact masses (MS) and fragmentation patters (MS/MS) of the peptides were collected in a data dependent manner utilizing dynamic exclusion to maximize depth of proteome coverage. Resulting peptide MS/MS spectral data were searched against the bacterial protein database using MaxQuant-LFQ along with subsequent MS1-based integrations and normalizations ${ }^{61}$. Statistical comparisons of resulting normalized protein quantitative values were performed using ProStaR ${ }^{62}$. Protein name, gene name, and subcellular localization of each identified peptide was manually determined using the UniProt and EcoCyc databases ${ }^{63,64}$.

\section{Ethidium bromide uptake assay}

Colony biofilms were grown at room temperature on YESCA agar. After 11 days, biofilms were homogenized by vortexing and sonication in PBS. After homogenization, a portion of the cellular fraction was removed and normalized to $\mathrm{OD}_{600}=0.5$ in PBS. Ethidium bromide (Bio-Rad) was added to a final concentration of $10 \mu \mathrm{g} / \mathrm{mL}$. Cells were then incubated at $37^{\circ} \mathrm{C}$ for $10 \mathrm{~min}$. Next, the suspensions were pelleted, supernatant removed, and cells were resuspended in $300 \mu \mathrm{L}$ PBS. Fluorescence of ethidium bromide was measured at $360 / 590 \mathrm{~nm}$. Each fluorescence measurement is the average of three technical replicates.

\section{PrestoBlue uptake assay}

Uptake of PrestoBlue (Invitrogen) was performed as previously described ${ }^{46}$. Planktonic cultures were grown to mid-logarithmic phase and normalized to $\mathrm{OD}_{600}=1.0$ in PBS. Biofilm cells were extracted from homogenized colony biofilms and normalized to $\mathrm{OD}_{600}=1.0$ in PBS. For each growth condition, $180 \mu \mathrm{L}$ of culture was mixed with $20 \mu \mathrm{L}$ PrestoBlue in a 96-well plate. Fluorescence at $560 / 590 \mathrm{~nm}$ was measured every five minutes for $1 \mathrm{~h}$. Each fluorescence measurement is the average of three technical replicates.

\section{Ethidium bromide efflux assay}

Efflux of ethidium bromide was performed as described previously ${ }^{47}$. Planktonic cultures were grown to mid-logarithmic phase, washed twice in PBS, and normalized to $\mathrm{OD}_{600}=0.5$. Biofilm cells were extracted from homogenized colony biofilms, washed twice with PBS, and normalized to $\mathrm{OD}_{600}=0.5$. Cells were loaded with ethidium bromide $(10 \mu \mathrm{g} / \mathrm{mL})$ in energy deplete conditions (PBS with $10 \mu \mathrm{g} / \mathrm{mL}$ proton ionophore carbonyl cyanide m-chlorophenyl hydrazone (CCCP)) for one hour at $37^{\circ} \mathrm{C}$. Cells were washed in PBS and resuspended in PBS $\pm 0.4 \% \mathrm{w} / \mathrm{v}$ glucose. Efflux was monitored by measuring fluorescence at $360 / 590 \mathrm{~nm}$ in technical triplicate at $37^{\circ} \mathrm{C}$ every minute for $15 \mathrm{~min}$.

\section{TTC reduction assays}

Triphenyl tetrazolium chloride (TTC) reduction assays were performed as described previously ${ }^{31}$. Ten microliter of overnight culture was spotted onto $1.2 \times$ YESCA agar containing $0.001 \%(w / v)$ TTC. After $24 \mathrm{~h}$ of growth, colonies were imaged using an Epson digital scanner. Images were subjected to automatic thresholding to subtract background, and TTC reduction was quantified by measuring pixel intensity on imageJ. Colony area was determined on Adobe Photoshop.

\section{ATP quantification}

ATP quantification was performed on cells extracted from homogenized colony biofilms grown on YESCA agar for 11 days. One aliquot of biofilm cells was removed, and ATP concentration was determined using the CellGlo Titer kit (Promega) according to manufacturer's protocols. Briefly, $50 \mu \mathrm{L}$ of bacterial suspension was mixed with an equal volume of Cell-Glo Titer reagent and incubated with shaking at room temperature for $15 \mathrm{~min}$. Luminescence was measured on a SpectraMax i3 plate reader (Molecular Devices) and converted to ATP concentration using a standard curve. A separate aliquot of the same sample was serially diluted for CFU enumeration. To account for differences in the number of cells between samples, ATP concentration was normalized to CFU per biofilm.

\section{Statistical analyses}

Statistical analyses were performed in GraphPad Prism. Details of sample size, test used, error bars, and statistical significance cutoffs are presented in the text or figure legends.

\section{Reporting summary}

Further information on research design is available in the Nature Research Reporting Summary linked to this article.

\section{DATA AVAILABILITY}

Proteomics data have been deposited in the Proteomics Identifications (PRIDE) Database (Accession: PXD023998). All remaining data can be found in the text and supplemental information, or will be made available upon request of the authors.

Received: 16 November 2020; Accepted: 16 March 2021; Published online: 16 April 2021

\section{REFERENCES}

1. Kostakioti, M., Hadjifrangiskou, M. \& Hultgren, S. J. Bacterial biofilms: development, dispersal, and therapeutic strategies in the dawn of the postantibiotic era. Cold Spring Harb. Perspect. Med. 3, a010306 (2013).

2. Flemming, H. C. et al. Biofilms: an emergent form of bacterial life. Nat. Rev. Microbiol. 14, 563-575 (2016). 
3. Stewart, P. S. \& Franklin, M. J. Physiological heterogeneity in biofilms. Nat. Rev. Microbiol. 6, 199-210 (2008).

4. Hall-Stoodley, L., Costerton, J. W. \& Stoodley, P. Bacterial biofilms: from the natural environment to infectious diseases. Nat. Rev. Microbiol. 2, 95-108 (2004).

5. Donlan, R. M. \& Costerton, J. W. Biofilms: survival mechanisms of clinically relevant microorganisms. Clin. Microbiol. Rev. 15, 167-193 (2002).

6. Costerton, J. W., Stewart, P. S. \& Greenberg, E. P. Bacterial biofilms: a common cause of persistent infections. Science 284, 1318-1322 (1999).

7. Vidakovic, L., Singh, P. K., Hartmann, R., Nadell, C. D. \& Drescher, K. Dynamic biofilm architecture confers individual and collective mechanisms of viral protection. Nat. Microbiol. 3, 26-31 (2018).

8. Flemming, H. C. \& Wuertz, S. Bacteria and archaea on Earth and their abundance in biofilms. Nat. Rev. Microbiol. 17, 247-260 (2019).

9. Mermel, L. A. et al. Clinical practice guidelines for the diagnosis and management of intravascular catheter-related infection: 2009 Update by the Infectious Diseases Society of America. Clin. Infect. Dis. 49, 1-45 (2009).

10. Floyd, K. A. et al. Adhesive fiber stratification in uropathogenic Escherichia coli biofilms unveils oxygen-mediated control of type 1 pili. PLoS Pathog. 11, e1004697 (2015)

11. Dragoš, A. et al. Division of labor during biofilm matrix production. Curr. Biol. 28 1903-1913.e1905 (2018).

12. Nadell, C. D., Drescher, K. \& Foster, K. R. Spatial structure, cooperation and competition in biofilms. Nat. Rev. Microbiol. 14, 589-600 (2016).

13. Klein, R. D. \& Hultgren, S. J. Urinary tract infections: microbial pathogenesis, hostpathogen interactions and new treatment strategies. Nat. Rev. Microbiol. 18, 211-226 (2020)

14. Flores-Mireles, A. L., Walker, J. N., Caparon, M. \& Hultgren, S. J. Urinary tract infections: epidemiology, mechanisms of infection and treatment options. Nat. Rev. Microbiol. 13, 269-284 (2015).

15. Foxman, B. The epidemiology of urinary tract infection. Nat. Rev. Urol. 7, 653-660 (2010).

16. Beebout, C. J. et al. Respiratory heterogeneity shapes biofilm formation and host colonization in uropathogenic Escherichia coli. mBio https://doi.org/10.1128/ mBio.02400-18 (2019).

17. Borisov, V \& Verkhovsky, M. Oxygen as acceptor. EcoSal Plus 6. https://doi.org/ 10.1128/ecosalplus.ESP-0012-2015 (2015).

18. Unden, G. \& Bongaerts, J. Alternative respiratory pathways of Escherichia coli: energetics and transcriptional regulation in response to electron acceptors. Biochim. Biophys. Acta 1320, 217-234 (1997).

19. Alteri, C. J., Smith, S. N. \& Mobley, H. L. Fitness of Escherichia coli during urinary tract infection requires gluconeogenesis and the TCA cycle. PLoS Pathog. 5, e1000448 (2009).

20. Hadjifrangiskou, M. et al. A central metabolic circuit controlled by QseC in pathogenic Escherichia coli. Mol. Microbiol. 80, 1516-1529 (2011).

21. Hadjifrangiskou, M. et al. Transposon mutagenesis identifies uropathogenic Escherichia coli biofilm factors. J. Bacteriol. 194, 6195-6205 (2012).

22. Floyd, K. A. et al. The Ubil ( $\mathrm{VisC}$ ) aerobic ubiquinone synthase is required for expression of type 1 pili, biofilm formation, and pathogenesis in uropathogenic Escherichia coli. J. Bacteriol. 198, 2662-2672 (2016).

23. Eberly, A. R. et al. Biofilm formation by uropathogenic Escherichia coli is favored under oxygen conditions that mimic the bladder environment. Int. J. Mol. Sci. https://doi.org/10.3390/ijms18102077 (2017).

24. Borisov, V. B., Gennis, R. B., Hemp, J. \& Verkhovsky, M. I. The cytochrome bd respiratory oxygen reductases. Biochim. Biophys. Acta 1807, 1398-1413 (2011).

25. Cotter, P. A., Melville, S. B., Albrecht, J. A. \& Gunsalus, R. P. Aerobic regulation of cytochrome d oxidase (cydAB) operon expression in Escherichia coli: roles of Fnr and ArcA in repression and activation. Mol. Microbiol. 25, 605-615 (1997).

26. Cotter, P. A. \& Gunsalus, R. P. Contribution of the fnr and arcA gene products in coordinate regulation of cytochrome $o$ and $d$ oxidase (cyoABCDE and cydAB) genes in Escherichia coli. FEMS Microbiol. Lett. 70, 31-36 (1992).

27. Cotter, P. A., Chepuri, V., Gennis, R. B. \& Gunsalus, R. P. Cytochrome o (cyoABCDE) and $d(c y d A B)$ oxidase gene expression in Escherichia coli is regulated by oxygen, $\mathrm{pH}$, and the fnr gene product. J. Bacteriol. 172, 6333-6338 (1990).

28. Giuffrè, A., Borisov, V. B., Arese, M., Sarti, P. \& Forte, E. Cytochrome bd oxidase and bacterial tolerance to oxidative and nitrosative stress. Biochim. Biophys. Acta 1837, 1178-1187 (2014).

29. Shepherd, M. et al. The cytochrome bd-I respiratory oxidase augments survival of multidrug-resistant Escherichia coli during infection. Sci. Rep. 6, 35285 (2016).

30. Mason, M. G. et al. Cytochrome bd confers nitric oxide resistance to Escherichia coli. Nat. Chem. Biol. 5, 94-96 (2009).

31. Jo, J., Cortez, K. L., Cornell, W. C., Price-Whelan, A. \& Dietrich, L. E. An orphan $c b b_{3^{-}}$ type cytochrome oxidase subunit supports Pseudomonas aeruginosa biofilm growth and virulence. elife https://doi.org/10.7554/eLife.30205 (2017).
32. Stewart, P. S. \& Parker, A. E. Measuring antimicrobial efficacy against biofilms: a meta-analysis. Antimicrob. Agents Chemother. https://doi.org/10.1128/AAC.0002019 (2019).

33. O'Toole, G. A. et al. Genetic approaches to study of biofilms. Methods Enzymol. 310, 91-109 (1999).

34. Kragh, K. N., Alhede, M., Kvich, L. \& Bjarnsholt, T. Into the well-a close look at the complex structures of a microtiter biofilm and the crystal violet assay. Biofilm https://doi.org/10.1016/j.bioflm.2019.100006 (2019).

35. Schiessl, K. T. et al. Phenazine production promotes antibiotic tolerance and metabolic heterogeneity in Pseudomonas aeruginosa biofilms. Nat. Commun. 10, 762 (2019).

36. Goneau, L. W. et al. Selective target inactivation rather than global metabolic dormancy causes antibiotic tolerance in uropathogens. Antimicrob. Agents Chemother. 58, 2089-2097 (2014).

37. CLSI. Performance Standards for Antimicrobial Susceptibility Testing. 30th ed. CLSI supplement M100. Wayne, PA: Clinical and Laboratory Standards Instititute; (2020).

38. Gupta, K. et al. International clinical practice guidelines for the treatment of acute uncomplicated cystitis and pyelonephritis in women: A 2010 update by the Infectious Diseases Society of America and the European Society for Microbiology and Infectious Diseases. Clin. Infect. Dis. 52, e103-e120 (2011).

39. Mironov, A. et al. CydDC functions as a cytoplasmic cystine reductase to sensitize. Proc. Natl Acad. Sci. USA https://doi.org/10.1073/pnas.2007817117 (2020).

40. Vadyvaloo, V. et al. Cell-surface alterations in class lla bacteriocin-resistant Listeria monocytogenes strains. Microbiology 150, 3025-3033 (2004).

41. McCrate, O. A., Zhou, X., Reichhardt, C. \& Cegelski, L. Sum of the parts: composition and architecture of the bacterial extracellular matrix. J. Mol. Biol. 425, 4286-4294 (2013).

42. Nikaido, $\mathrm{H}$. Molecular basis of bacterial outer membrane permeability revisited Microbiol. Mol. Biol. Rev. 67, 593-656 (2003).

43. Delcour, A. H. Outer membrane permeability and antibiotic resistance. Biochim Biophys. Acta 1794, 808-816 (2009).

44. Nikaido, H. \& Pagès, J. M. Broad-specificity efflux pumps and their role in multidrug resistance of Gram-negative bacteria. FEMS Microbiol. Rev. 36, 340-363 (2012)

45. Schembri, M. A., Kjaergaard, K. \& Klemm, P. Global gene expression in Escherichia coli biofilms. Mol. Microbiol. 48, 253-267 (2003).

46. Bhattacharyya, S., Walker, D. M. \& Harshey, R. M. Dead cells release a 'necrosignal' that activates antibiotic survival pathways in bacterial swarms. Nat. Commun. 11, 4157 (2020).

47. Paixão, L. et al. Fluorometric determination of ethidium bromide efflux kinetics in Escherichia coli. J. Biol. Eng. 3, 18 (2009).

48. Du, D. et al. Multidrug efflux pumps: structure, function and regulation. Nat. Rev. Microbiol. 16, 523-539 (2018).

49. Sielaff, H., Duncan, T. M. \& Börsch, M. The regulatory subunit $\varepsilon$ in Escherichia coli F. Biochim. Biophys. Acta Bioenerg. 1859, 775-788 (2018).

50. Pagès, J. M., James, C. E. \& Winterhalter, M. The porin and the permeating antibiotic: a selective diffusion barrier in Gram-negative bacteria. Nat. Rev. Microbiol. 6, 893-903 (2008).

51. Proctor, R. A. et al. Small colony variants: a pathogenic form of bacteria that facilitates persistent and recurrent infections. Nat. Rev. Microbiol. 4, 295-305 (2006).

52. Bajeli, S. et al. Terminal respiratory oxidases: a targetables vulnerability of mycobacterial bioenergetics? Front. Cell Infect. Microbiol. 10, 589318 (2020).

53. Lee, B. S. et al. Dual inhibition of the terminal oxidases eradicates antibiotictolerant Mycobacterium tuberculosis. EMBO Mol. Med. https://doi.org/10.15252/ emmm.202013207 (2020).

54. Conradie, F. et al. Treatment of highly drug-resistant pulmonary tuberculosis. $N$. Engl. J. Med. 382, 893-902 (2020).

55. de Jager, V. R. et al. Telacebec (Q203), a New Antituberculosis Agent. N. Engl. J. Med. 382, 1280-1281 (2020).

56. Mogi, T. et al. Antibiotics LL-Z1272 identified as novel inhibitors discriminating bacterial and mitochondrial quinol oxidases. Biochim. Biophys. Acta 1787, 129-133 (2009)

57. Mulvey, M. A., Schilling, J. D. \& Hultgren, S. J. Establishment of a persistent Escherichia coli reservoir during the acute phase of a bladder infection. Infect. Immun. 69, 4572-4579 (2001).

58. Murphy, K. C. \& Campellone, K. G. Lambda Red-mediated recombinogenic engineering of enterohemorrhagic and enteropathogenic E. coli. BMC Mol. Biol. 4 11 (2003).

59. Wiegand, I., Hilpert, K. \& Hancock, R. E. Agar and broth dilution methods to determine the minimal inhibitory concentration (MIC) of antimicrobial substances. Nat. Protoc. 3, 163-175 (2008).

60. Pfaffl, M. W. A new mathematical model for relative quantification in real-time RTPCR. Nucleic Acids Res. 29, e45 (2001). 
12

61. Cox, J. et al. Accurate proteome-wide label-free quantification by delayed normalization and maximal peptide ratio extraction, termed MaxLFQ. Mol. Cell Proteomics 13, 2513-2526 (2014).

62. Wieczorek, S. et al. DAPAR \& ProStaR: software to perform statistical analyses in quantitative discovery proteomics. Bioinformatics 33, 135-136 (2017).

63. Keseler, I. M. et al. The EcoCyc database: reflecting new knowledge about Escherichia coli K-12. Nucleic Acids Res. 45, D543-D550 (2017).

64. Consortium, U. UniProt: a worldwide hub of protein knowledge. Nucleic Acids Res. 47, D506-D515 (2019).

\section{ACKNOWLEDGEMENTS}

We thank members of the Hadjifrangiskou and Schmitz labs for helpful discussions and critical reading of the manuscript. Proteomics studies were performed at the Vanderbilt Mass Spectrometry Research Center with the help of W. Hayes McDonald, and microscopy was performed at the Vanderbilt Cell Imaging Shared Resource. This work was supported by the National Institutes of Health under the following grants: R01Al107052 (M.H.), P20DK123967 (M.H.), T32GM007347 (C.J.B.), and F30Al150077 (C.J.B.).

\section{AUTHOR CONTRIBUTIONS}

C.J.B. and M.H. conceived the study, performed the experiments, analyzed the data, and composed the manuscript. L.A.S., A.R.E., and G.T.V.H. performed the experiments, analyzed the data, and edited the manuscript.

\section{COMPETING INTERESTS}

The authors declare no competing interests.

\section{ADDITIONAL INFORMATION}

Supplementary information The online version contains supplementary material available at https://doi.org/10.1038/s41522-021-00210-x.

Correspondence and requests for materials should be addressed to M.H.

Reprints and permission information is available at http://www.nature.com/ reprints

Publisher's note Springer Nature remains neutral with regard to jurisdictional claims in published maps and institutional affiliations.

(c)

Open Access This article is licensed under a Creative Commons Attribution 4.0 International License, which permits use, sharing, adaptation, distribution and reproduction in any medium or format, as long as you give appropriate credit to the original author(s) and the source, provide a link to the Creative Commons license, and indicate if changes were made. The images or other third party material in this article are included in the article's Creative Commons license, unless indicated otherwise in a credit line to the material. If material is not included in the article's Creative Commons license and your intended use is not permitted by statutory regulation or exceeds the permitted use, you will need to obtain permission directly from the copyright holder. To view a copy of this license, visit http://creativecommons. org/licenses/by/4.0/.

(c) The Author(s) 2021 